\title{
Maintained Expression of the Planar Cell Polarity Molecule Vangl2 and Reformation of Hair Cell Orientation in the Regenerating Inner Ear
}

\author{
Mark E. Warchol ${ }^{1}$ and Mireille Montcouquiol ${ }^{2}$ \\ ${ }^{1}$ Fay and Carl Simons Center for the Biology of Hearing and Deafness, Department of Otolaryngology, Washington University \\ School of Medicine, P.O. Box 8115, 660 South Euclid Ave., St. Louis, MO 63110, USA \\ ${ }^{2}$ INSERM U862, Neurocentre Magendie, University of Bordeaux II, Bordeaux, 3300, France
}

Received: 4 May 2009; Accepted: 18 January 2010; Online publication: 23 February 2010

\begin{abstract}
The avian inner ear possesses a remarkable ability to regenerate sensory hair cells after ototoxic injury. Regenerated hair cells possess phenotypes and innervation that are similar to those found in the undamaged ear, but little is known about the signaling pathways that guide hair cell differentiation during the regenerative process. The aim of the present study was to examine the factors that specify the orientation of hair cell stereocilia bundles during regeneration. Using organ cultures of the chick utricle, we show that hair cells are properly oriented after having regenerated entirely in vitro and that orientation is not affected by surgical removal of the striolar reversal zone. These results suggest that the orientation of regenerating stereocilia is not guided by the release of a diffusible morphogen from the striolar reversal zone but is specified locally within the regenerating sensory organ. In order to determine the nature of the reorientation cues, we examined the expression patterns of the core planar cell polarity molecule Vang12 in the normal and regenerating utricle. We found that Vangl2 is asymmetrically expressed on cells within the sensory epithelium and that this expression pattern is maintained after ototoxic injury and throughout regeneration. Notably, treatment with a small molecule inhibitor of c-Jun-N-terminal kinase disrupted the orientation of regenerated hair cells. Both of these results are consistent with the
\end{abstract}

Correspondence to: Mark E. Warchol - Fay and Carl Simons Center for the Biology of Hearing and Deafness, Department of Otolaryngology - Washington University School of Medicine · P.O. Box 8115, 660 South Euclid Ave., St. Louis, MO 63110, USA. Telephone: +1-3147477186; fax: +1-314-7477230; email: warcholm@ent.wustl.edu hypothesis that noncanonical Wnt signaling guides hair cell orientation during regeneration.

Keywords: vestibular, regeneration, development, stereocilia, planar cell polarity, hair cell, utricle

\section{INTRODUCTION}

Sensory hair cells are the specialized mechanoreceptors of the cochlea and vestibular organs and are responsible for the detection of sound vibrations and head movements. All vertebrate hair cells display certain commonalities in their structure and function, but many morphological features of these cells (e.g., stereocilia number, height, and orientation) can vary, depending upon a cell's position within a particular sensory epithelium. In the vestibular organs, a hair cell's orientation is a key determinant of its directional response to head movements. Hair cells in the cristae of the semicircular canals are uniformly orientated and display similar responses (i.e., either depolarization or hyperpolarization) to a given rotational stimulus. In contrast, hair cells in the otolithic maculae are divided into two populations with opposing orientations, so that an arbitrary linear acceleration or head tilt will evoke membrane depolarization in one group of macular hair cells and hyperpolarization in the other group. Such morphological features have important consequences for the coding of sensory information that is transmitted from the periphery to the vestibular brainstem nuclei (e.g., Lysakowski and Goldberg 2005). 
One notable feature of the avian inner ear is that its hair cells can quickly regenerate after ototoxic injury, leading to a nearly complete restoration of sensory function (e.g., Matsui et al. 2005). In the vestibular organs, regenerated hair cells reacquire the same orientations and physiological sensitivities as their counterparts in the undamaged ear (Dye et al. 1999; Zakir and Dickman 2006), but the molecular signals that regulate hair cell structure and orientation during regeneration are not known. Recent developmental studies of the mammalian ear have demonstrated that molecules of the planar cell polarity (PCP) pathway play a critical role in the establishment of hair cell orientation (Montcouquiol et al. 2003, 2006a; Wang et al. 2006; Deans et al. 2007). Expression of certain PCP pathway molecules has also been observed during the development of the avian ear (Davies et al. 2005), but very little is known about the role of these molecules during regeneration. In the present study, we have used organ cultures of the chick utricle to examine the mechanisms governing hair cell orientation during regeneration. We report that recovery of stereocilia orientation does not require signaling from the striolar reversal zone but appears instead to be specified locally within the regenerating epithelium. We also observed that the core PCP molecule Vang12 continues to be expressed in the mature utricle and that its expression is maintained after ototoxic injury and throughout regeneration. Finally, inhibition of c-Jun-N-terminal kinase (JNK) signaling resulted in disorientation of regenerated stereocilia. These results are consistent with a proposed role for noncanonical Wnt signaling in the specification of stereocilia orientation during regeneration.

\section{METHODS}

\section{Animals}

Fertile chicken eggs (White Leghorn strain) were obtained from Charles River (Franklin, CT, USA) and incubated at $37^{\circ} \mathrm{C}$ in a humidified environment. Hatchling chicks were maintained in heated brooders for 23 weeks prior to use. All experimental protocols involving animals were approved by the Washington University Institutional Animal Care and Use Committee and conformed to the National Institutes of Health animal use guidelines.

\section{Organ culture techniques}

Organ cultures of the chick utricle were prepared following previously published protocols (Warchol 1999; Warchol and Speck 2007). Chickens (14-21 days post-hatch) were euthanized with $\mathrm{CO}_{2}$ and decapitated. Following removal of the lower jaw and skin, the heads were immersed in $70 \%$ ethanol for $\sim 5-10 \mathrm{~min}$, in order to kill surface pathogens. The membranous labyrinth was exposed, and a section of the temporal bone (including the utricle) was transferred to chilled dissection medium (Medium-199 containing Hank's salts and HEPES buffer (Invitrogen)). Utricles were then isolated from the temporal bone explants, and the otoconia and otolithic membranes were removed using fine forceps. Individual utricles were transferred to culture wells (Mat Tek, Ashland, MA, USA) that contained $100 \mu \mathrm{l}$ of medium (Medium-199 supplemented with Earle's salts, 2,200 mg/L sodium bicarbonate, $0.69 \mathrm{mM}$ L-glutamine, $25 \mathrm{mM}$ HEPES, and $2.5 \%$ fetal bovine serum (Invitrogen)). Streptomycin sulfate (Sigma) was also added to the culture wells, for a final concentration of $1 \mathrm{mM}$. Cultures were incubated in streptomycin-containing medium for $24 \mathrm{~h}$ at $37^{\circ} \mathrm{C}$ in a humidified $5 \% \mathrm{CO}_{2} / 95 \%$ air environment. At this point, cultures were rinsed $3 \times$ in fresh medium and maintained in streptomycin-free medium for an additional 1-7 days. Cultures were fed fresh medium every 2-3 days.

\section{Treatment with small molecule inhibitors}

In order to investigate the possible roles of JNK and Erk1/2 in the process of hair cell orientation, small molecule inhibitors of these kinases were added to organ cultures. Results of prior studies suggest that cell proliferation in our organotypic culture system peaks at about $48 \mathrm{~h}$ after streptomycin treatment, and then returns to baseline levels at 4 days post-streptomycin (Matsui et al. 2000). In order to specifically examine the effects of JNK and Erk1/2 inhibition on cell differentiation (and to avoid any confounding effects on cell proliferation), the inhibitors were added to cultures beginning at 4 days after completion of streptomycin treatment. Cultures were then treated for 3 days with either SP600125 (JNK inhibitor, $15 \mu \mathrm{M}$; Calbiochem/EMD Biosciences) or U0126 (Erk1/2/inhibitor, $10 \mu \mathrm{M}$; Calbiochem/EMD Biosciences). Stock solutions were prepared in DMSO, and concentrated aliquots were stored at $-20^{\circ} \mathrm{C}$ for $<30$ days. All control specimens in these experiments were treated with $0.1 \%$ DMSO.

\section{Tissue processing and immunohistochemistry}

Specimens were fixed for $20 \mathrm{~min}$ with $4 \%$ paraformaldehyde in $0.1 \mathrm{M}$ phosphate buffer $(\mathrm{pH}=7.4)$ and were then rinsed $5 \times$ with $0.01 \mathrm{M}$ phosphate buffered saline (PBS; Sigma). Nonspecific antibody binding was blocked by treatment for $2 \mathrm{~h}$ in $5 \%$ normal horse serum in PBS, with $0.2 \%$ Triton X-100. Specimens were incubated in primary antibodies for 16-24 h (at $4^{\circ} \mathrm{C}$ ) and were then rinsed and treated for $2 \mathrm{~h}$ in secondary antibodies (at room temperature). Second- 
aries (anti-mouse or anti-rabbit) were conjugated to either Cy3 (Jackson Research) or Alexa-488 (Molecular Probes). Nuclear DNA was labeled with DAPI. After labeling, specimens were placed upright on glass microscope slides in a drop of glycerol:PBS (9:1) and cover-slipped.

\section{Background data on primary antibodies}

Vestibular hair cells were labeled with a rabbit polyclonal antibody raised against calretinin (Ab-1, Oncogene Sciences; diluted 1:500). Acetylated tubulin was labeled with a mouse monoclonal raised against acetylated $\alpha$-tubulin from sea urchin (clone 611B-1; Sigma; 1:500), and alpha-spectrin was labeled with a mouse monoclonal raised against chick red blood cells (Chemicon/Millipore, 1:100). The core planar polarity molecule Vangl2 was labeled using a customraised rabbit polyclonal, as described in Montcouquiol et al. (2006a). Filamentous actin was labeled with Alexa-488-phalloidin (Molecular Probes/Invitrogen, in PBS $/ 0.2 \%$ Triton X-100).

\section{Imaging and data analysis}

Specimens were imaged on inverted microscopes (Zeiss Axiovert 135), using epifluorescence illumination. Images were obtained with a cooled CCD camera (Photonics Cool Snap ES, Roper Scientific, Tucson, AZ, USA) and colorized and sharpened using IP Lab (Scanalytics, Fairfax, VA, USA) software. Quantification was carried-out directly from stored images, and final cell densities were normalized to $10,000 \mu \mathrm{m}^{2}$ fields. Confocal images were obtained with a Bio-Rad Radiance 2000 MP system, using a Nikon Eclipse inverted microscope. Image stacks were assembled with Volocity software and processed with Adobe Photoshop. Statistical tests were performed with Microsoft Excel. All quantitative data are expressed and plotted as mean \pm SD. Finalized figures were assembled with Adobe Photoshop and Adobe Illustrator.

\section{RESULTS}

Loss and regeneration of stereocilia following streptomycin treatment

Initial experiments characterized ototoxic injury and regeneration in the chick utricle, using a culture system that has been described elsewhere (e.g., Matsui et al. 2000; Warchol and Richardson 2009). Briefly, chick utricles were placed in organ culture and maintained for $24 \mathrm{~h}$ in medium that contained $1 \mathrm{mM}$ streptomycin (in order to lesion hair cells). The cultures were then thoroughly rinsed and maintained for an additional 17 days in streptomycin-free medium. Fixed specimens were labeled with Alexa-488-conjugated phalloidin (to identify stereocilia) and anti-calretinin (to identify hair cells). After processing, the numbers of surviving stereocilia bundles and hair cells were imaged and quantified. Utricles that were fixed at 1 day after streptomycin treatment contained $7.2 \pm 6.0$ stereocilia bundles/ $10,000 \mu \mathrm{m}^{2}$ and $8.6 \pm 5.1$ hair cells $/ 10,000 \mu \mathrm{m}^{2}$ (mean \pm $\mathrm{SD}, n=25$ samples from eight specimens; Fig. 1B). Since hair cell density in the uninjured chick utricle is $\sim 180$ / $10,000 \mu \mathrm{m}^{2}$ (Warchol 2001), placement in organ culture and treatment with streptomycin resulted in the loss of about $96 \%$ of the total hair cell population. In contrast, utricles that were allowed to recover in vitro for 7 days after streptomycin treatment contained $42.3 \pm 14.1$ stereocilia bundles/10,000 $\mu \mathrm{m}(n=37$ samples from nine specimens; Fig. 1C). Based on previous data (Matsui et al. 2000; Warchol and Richardson 2009), we attribute this sixfold increase in stereocilia bundles to hair cell regeneration in vitro.

\section{Correct stereocilia alignment occurs after regeneration in vitro}

One notable observation of in vivo studies of regeneration in the avian maculae is that recovered hair cells are correctly oriented with respect to the striolar reversal zone (e.g., Dye et al. 1999). In order to determine whether correct orientation also occurs after hair cell injury and regeneration in vitro, we treated utricles $(n=$ 10) with streptomycin and then allowed them to regenerate for 7 days. Fixed specimens were immunolabeled for acetylated tubulin, which labels the kinocilia and apical surfaces of regenerated hair cells (Fig. 2C). Specimens were examined as whole-mounts and positioned so that their lateral-medial axis was oriented along the horizontal $(x)$ axis of the image field. In addition, the lateral (striolar) edge of the specimens was placed at the position corresponding to $180^{\circ}$ in the coordinate scheme shown in Figure 2(A, B). (Note: The diagrams and coordinates shown in Figs. 2 and 4 correspond to data from the right utricle. Orientation data from left-side specimens were collected using a mirror image of the illustrated coordinates, so that the resulting angular data are comparable.) High magnification images of hair cell surfaces were obtained from throughout the medial extrastriolar region of the utricle, and the angular orientations of kinocilia (relative to medial pole) were quantified using IP Lab software (Fig. 2B). Hair cell kinocilia in the medial region of the utricle are normally positioned near the lateral surface of the hair cell (e.g., Fig. 2B, C), so that stimuli directed toward the striolar reversal zone will result in membrane depolarization. Data were obtained from a total of 364 bundles from ten specimens. Individual orientations were plotted as a histogram (Fig. 2D) and yielded a mean orientation of $179.8 \pm$ 

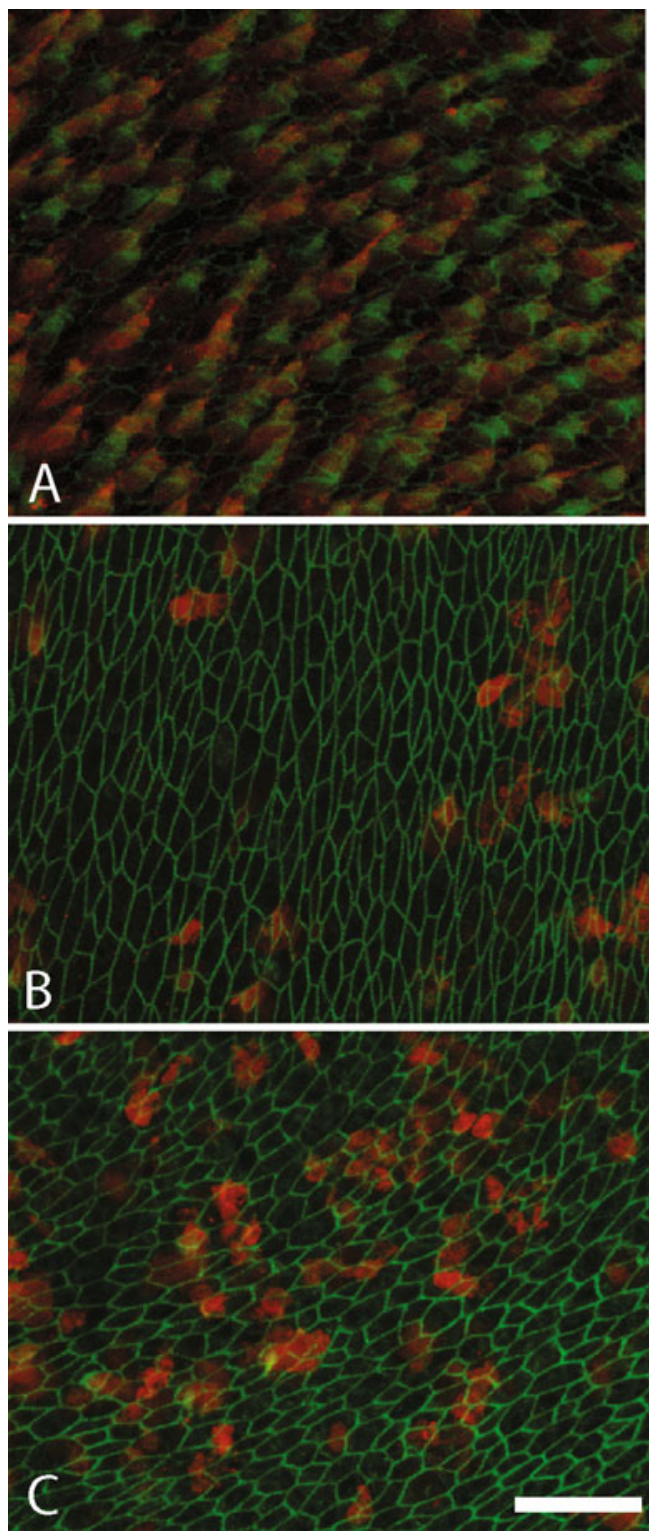

FIG. 1. Ototoxic injury and regeneration in organotypic cultures of the chick utricle. Hair cells are immunolabeled for calretinin (red), and stereocilia bundles and cell-cell junctions are labeled with phalloidin (green). A Numerous hair cells (red) were present in utricles that were cultured for 8 days in normal medium. B Treatment for $24 \mathrm{~h}$ with $1 \mathrm{mM}$ streptomycin caused a dramatic reduction in the numbers of labeled hair cells (red) and stereocilia bundles. C After 7 days recovery from streptomycin injury, numerous regenerated hair cells (red) were evident. Scale bar $=20 \mu \mathrm{m}$.

$46.1^{\circ}$ (mean $\left.\pm \mathrm{SD}\right)$. Although the mean orientation is very near the expected value of $180^{\circ}$, the histogram revealed a biphasic distribution of orientations, with clusters around $\sim 150^{\circ}$ and $210^{\circ}$. This outcome is probably a consequence of the fact that the reversal zone is not strictly parallel to the vertical axis of the coordinate system that was used in these measurements (see Fig. 2A).
Removal of the striolar region does not affect the orientation of regenerating bundles

How is correct bundle orientation re-established during regeneration? One possibility is that a secreted morphogen is released from the striolar reversal zone, which then directs the bundles of regrowing hair cells to orient towards the striola. If this were correct, then removing the striolar region prior to regeneration should result in misalignment of regenerated stereocilia. In order to test this prediction, we surgically removed the striolar reversal zone from regenerating utricles and then quantified the orientation of regenerated stereocilia. Iridectomy scissors were used to excise the lateral region of the utricle (including the reversal zone), either just before $(n=8)$ or immediately after $(n=16)$ streptomycin treatment (Fig. 3). These striola-free specimens (which still contained about $70 \%$ of the sensory region of the utricle) were allowed to regenerate for 7 days in organ culture. At this time, they were fixed and processed for acetylated-tubulin immunohistochemistry. Since the lesions caused minor folding of the anterior and posterior portions of the sensory epithelium, images were obtained from middle $\sim 75 \%$ of the utricles. The orientation of individual stereocilia within this region was quantified, as described above. Data obtained from 368 bundles (from eight specimens) yielded a mean bundle orientation of $195.2 \pm 29.1^{\circ}$. As was observed in the intact utricle (Fig. 2D), a histogram plot of these data shows a bimodal distribution of orientations that is centered around $\sim 180^{\circ}$ (Fig. 4C). Notably, we observed slightly less variability in the stereociliary orientations in the lesioned utricles vs. those in the controls (compare Fig. 2D vs. Fig. 4C); we attribute this apparent difference to the smaller sampling areas that were analyzed in the lesioned utricles (see above). We also examined the orientation of regenerated stereocilia bundles in portions of utricles $(n=4)$ that were cut into halves along the lateral-medial axis (i.e., perpendicular to the striola) immediately after streptomycin treatment; hair cell orientation in those specimens appeared normal (data not shown). Taken together, these observations demonstrate that regenerated hair cells in the medial extrastriolar region of the utricle can still develop correctly oriented stereocilia bundles in the absence of active signaling from the striolar reversal zone.

Maintained expression of the core PCP molecule Vangl2 in the mature utricle

Hair cell orientation in the developing mammalian ear is dependent upon the proper expression of molecules of the PCP pathway (Montcouquiol et al. 2003, 2006a; Wang et al. 2006; Deans et al. 2007). In particular, genetic disruption of Vangl2 (the vertebrate orthologue of strabismus - Wolff and Rubin 1998) 
A
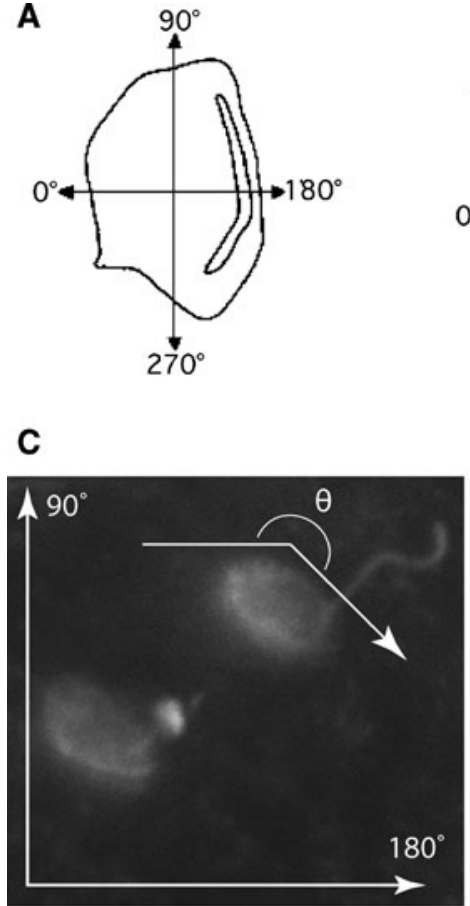

FIG. 2. Quantification of the orientation of regenerated stereocilia bundles. Specimens were labeled with an antibody against acetylated tubulin, which labeled hair cell apical surfaces and kinocilia. Utricles were then imaged on an epifluoresence microscope and positioned so that their lateral-medial axis was aligned along the horizontal axis of the visual field, yielding the coordinate system shown in (A). Planar polarity was quantified from high magnification images of immunolabeled hair cells, following a method that is shown schematically in (B). Specifically, we used image analysis
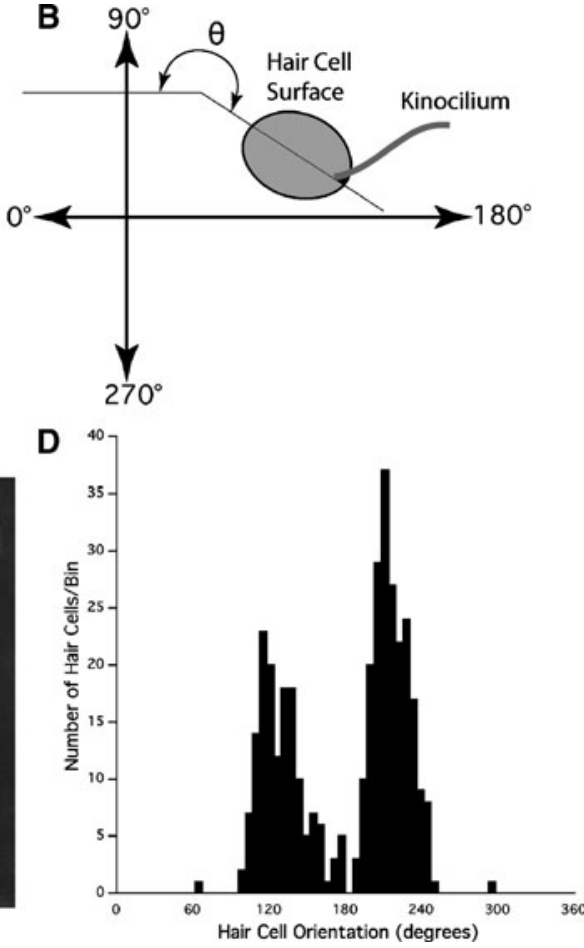

software to quantify the angular position of the kinocilium (labeled as " $\theta$ " in $\mathbf{B}$ and $\mathbf{C}$ ) on the apical surfaces of individual hair cells. An example of an actual orientation measurement is shown in (C). Orientation data were obtained from 364 hair cells, and the distribution of those orientations is shown in the histogram in (D). Note that the orientations were clustered around $180^{\circ}$, with nodes at $\sim 150^{\circ}$ and $210^{\circ}$. Those date indicate that correct stereocilia polarity is re-established after injury and regeneration in vitro.

in the extrastriolar region - Fig. 5C, D). We also used an antibody against $\alpha$-spectrin (which stains the entire apical surface of hair cells except for the insertion point of kinocilia-e.g., Deans et al. 2007) to examine the possible changes in the cellular localization of Vangl2 on opposing sides of the reversal line. Immunolabeling for Vangl2 was observed on cell junctions that were adjacent to both the excitatory (kinociliary) and inhibitory faces of hair cells on each side of the reversal line (Fig. 5E, F), but it was not possible to determine whether Vangl2 was expressed by hair cells or by adjoining supporting cells.

\section{Patterns of Vangl2 expression are unchanged after ototoxic injury}

In order to determine whether expression of core PCP molecules might be capable of providing orientation cues during regeneration, we next examined immunoreactivity for Vangl2 after ototoxic injury and during regeneration. As described above (e.g., Fig. 1), culture for $24 \mathrm{~h}$ in $1-\mathrm{mM}$ streptomycin resulted in a large-scale loss of utricular hair cells. Notably, Vang12 cell junctions that were oriented perpendicular to the reversal zone (i.e., shifted $\sim 90^{\circ}$ from labeling observed 


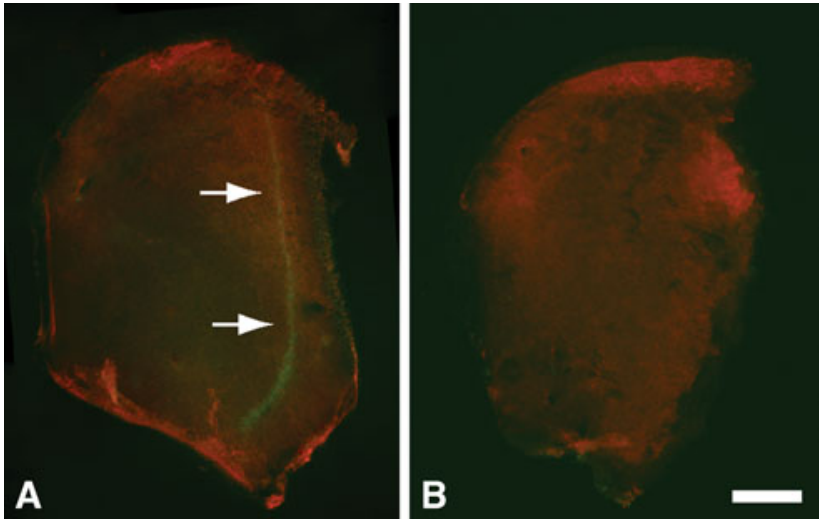

FIG. 3. Surgical removal of the reversal zone from cultured utricles. A In the normal utricle, the reversal zone can be identified by immunoreactivity for GATA3 (green, arrows). B This region can be excised from the utricle (using iridectomy scissors) prior to ototoxic injury and regeneration. Scale bar $=200 \mu \mathrm{m}$.

continued to be expressed in a normal polarized fashion in the remaining supporting cells (Fig. 6). As in undamaged specimens, immunoreactivity for Vangl2 in the extrastriolar region was largely confined to lateral and/or medial cell-cell junctions (Fig. 6A, B), while cells in the reversal zone frequently displayed labeling on their anterior and/or posterior faces (Fig. 6C, D). These expression patterns continued throughout the regenerative process; after 5 and 7 days of recovery, the patterns of Vang12 expression appeared essentially unchanged (Fig. 7). These observations demonstrate that supporting cells continue to express Vangl2 in a polarized fashion after ototoxic injury and during hair cell recovery.

\section{Inhibition of JNK disrupts the orientation of regenerated stereocilia bundles}

Interaction between core PCP molecules initiates signaling within the noncanonical Wnt pathway and ultimately leads to activation of the JNK (reviewed by Montcouquiol et al. 2006b; Jones and Chen 2006). Since interfering with JNK activation can cause downstream disruption of PCP signaling (e.g., Kim and Han 2005), we next examined whether JNK activation was necessary for the correct orientation of regenerated hair cells. As a control, we also examined the orientation of regenerated hair cells following inhibition of the extracellular signal-regulated kinases 1/2 (Erk1/2). Both JNK and Erk1/2 are members of the mitogenactivated protein kinase superfamily, but Erk1/2 is not thought to be essential for PCP signaling. Utricles $(n=$ 18) were cultured in $1 \mathrm{mM}$ streptomycin for $24 \mathrm{~h}$ (as above), followed by $96 \mathrm{~h}$ in normal medium. Specimens were then maintained for $72 \mathrm{~h}$ in medium that contained either $15 \mu \mathrm{M}$ SP600125 (a specific inhibitor of JNK-Bennett et al. 2001; $n=10$ ) or $10 \mu \mathrm{M} \mathrm{U0126}$ (an inhibitor of Erk1/2-Favata et al. 1998; $n=8$ ). Fixed utricles were processed for the visualization of acetylated tubulin, and hair cell polarization was quantified. The mean angular orientation of hair cells in JNK-inhibited cultures was $176.5 \pm 91.0^{\circ}$ (mean \pm SD, $n=223$ cell from ten specimens). Examination of the corresponding histogram plot (Fig. 8A), combined with the high standard deviation of bundle orientation $\left( \pm 91^{\circ}\right)$, suggests that SP600125 treatment resulted in a nearly complete disruption of hair cell orientation. In contrast, the mean orientation of hair cells after U0126 treatment was $184.6 \pm 41.8^{\circ} \quad(n=264)$, which is almost identical to value that was observed in control utricles $\left(179.8 \pm 46.1^{\circ}\right)$. Moreover, the distribution of orientations in both the U0126-treated and control specimens was bimodal and centered around $180^{\circ}$, with peaks at $\sim 150^{\circ}$ and $\sim 210^{\circ}$ (Figs. $2 \mathrm{C}$ and $8 \mathrm{~B}$ ). Still, it is noteworthy that the distribution of bundle orientation in the U0126-treated specimens was slightly different from that derived from control utricles (compare Fig. 2C with Fig. $8 \mathrm{~B}$ ) of the regenerated stereocilia bundles.

\section{Inhibition of JNK does not disrupt} the asymmetrical localization of Vangl2

In noncanonical Wnt signaling, activation of JNK occurs downstream of interactions between core PCP molecules (Boutros et al. 1998). Thus, even though JNK inhibition disrupts hair cell orientation, it should have no effect on the patterned expression of core PCP molecules. In order to test this prediction, we examined the localization of Vangl2 in regenerating utricles that had been treated for recovery days 5-7 with the JNK inhibitor SP600125 (as above). Consistent with our prediction, JNK inhibition did not alter the localization of Vangl2 in the sensory region of the utricle (Fig. 8C, D).

\section{DISCUSSION}

Hair cells in the avian cochlea and vestibular organs can regenerate after injury, leading to morphological and functional recovery. Numerous studies have demonstrated that regenerated hair cells possess similar phenotypes, orientations, and innervation as their counterparts in undamaged sensory organs, but relatively little is known about the signals that regulate pattern formation during regeneration. Results from the present study demonstrate that normal hair cell orientation is re-established after regeneration in organ culture and does not require chemical signaling from the striolar reversal zone. We further show that the core PCP molecule Vangl2 is asymmetrically expressed on utricular supporting cells and that this expression pattern is maintained after ototoxic injury 


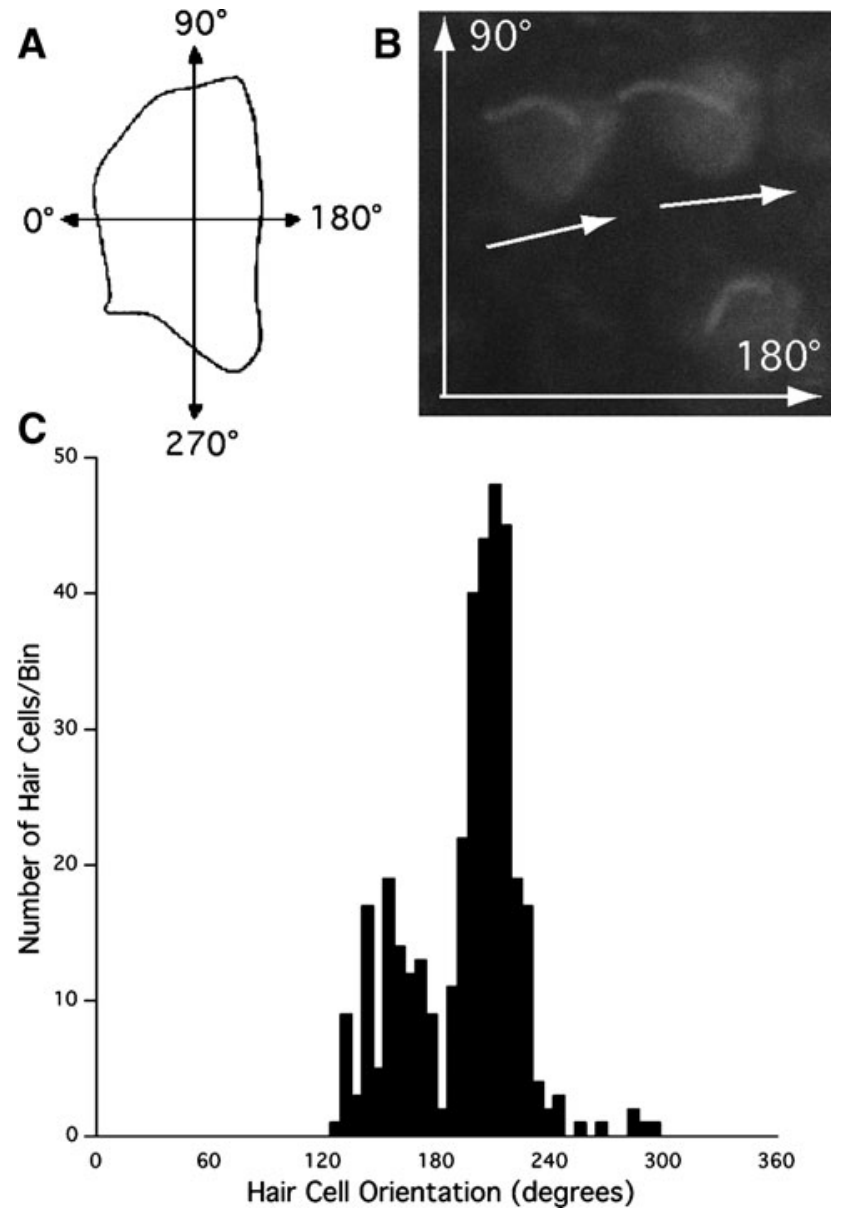

FIG. 4. Orientation of regenerated hair cells after surgical removal of the reversal zone. Quantitative data on hair cell orientation were obtained from 368 hair cells that had regenerated after striola removal ( $n=8$ utricles). The coordinate system and quantification method were identical to those shown in Figure 2. The angular positions of labeled kinocilia were quantified (e.g., axes and arrows in B), and a histogram of the resulting orientations (C) closely resembled the distribution observed from hair cells in intact utricles (e.g., Fig. 2D). Specifically, distribution of hair cell orientations was bimodal and clustered around $180^{\circ}$. These data suggest that the recovery of hair cell orientation does not depend on signaling from the striolar region.

and during regeneration. This finding suggests that signaling via core PCP molecules may specify hair cell polarity during regeneration. Consistent with this suggestion, we show that inhibition of JNK (a downstream signal in the PCP pathway) causes regenerated bundles to become disoriented.

\section{Orientation of regenerating stereocilia bundles in vitro}

The avian inner ear has become a widely studied model of vertebrate regeneration (e.g., Stone and Cotanche 2007). During regeneration, the majority of replacement hair cells are created via the renewed proliferation of epithelial supporting cells (Weisleder and Rubel 1993). Since morphological studies have demonstrated that the orientation of regenerated stereocilia bundles is nearly normal (e.g., Dye et al. 1999), it is clear that the vestibular organs of the mature birds retain whatever signaling molecules are necessary for the re-establishment of correct planar polarity. Nevertheless, the nature of those guidance signals has remained uncertain. It is possible, for example, that cell polarity is influenced by a secreted morphogen that is released from either a discrete region within the sensory epithelium or from a locus outside of the sensory organ. In this model, the putative morphogen would act as a chemo-attractant, to orient regrowing stereocilia. The observation that diffusible Wnt ligands can influence the orientation of stereocilia in the embryonic cochlea (e.g., Dabdoub et al. 2003; Dabdoub and Kelley 2005) provides support for the presence of such a mechanism during inner ear development. The results presented here, however, suggest that specification of cell polarity during regeneration is not regulated by a process of this type. Instead, we show that the orientation of regenerated hair cells in cultured utricles is essentially normal, implying that whatever cellular signals are necessary for proper orientation are contained within the sensory organs themselves. Moreover, we observed normal orientation of regenerated hair cells in the medial extrastriolar region of the utricle (known as the cotillus-Jørgensen 1989), even after surgical removal of the striolar reversal zone prior to regeneration. This result indicates that the release of a diffusible morphogen from the reversal zone is not necessary for guiding the orientation of new stereocilia. Instead, our results are most compatible with the notion that information on cell directionality is preserved locally within the injured sensory epithelium.

Expression of Vangl2 and the preservation of cell polarity

Given that recovery of stereocilia orientation does not require exogenous signaling, how do cells in the sensory epithelium "remember" their correct polarity after ototoxic injury and during regeneration? Studies of inner ear development have suggested a critical role for core PCP molecules in the alignment of stereocilia. Specifically, normal expression of the core PCP molecule Vangl2 is necessary for proper orientation of stereocilia in both the cochlea and vestibular organs (Montcouquiol et al. 2003, 2006a; Wang et al. 2006). Polarized expression of c-Fli1 (the chicken orthologue of flamingo, an atypical cadherin and core PCP molecule) has also been described in the developing chick ear (Davies et al. 2005). The present study is 

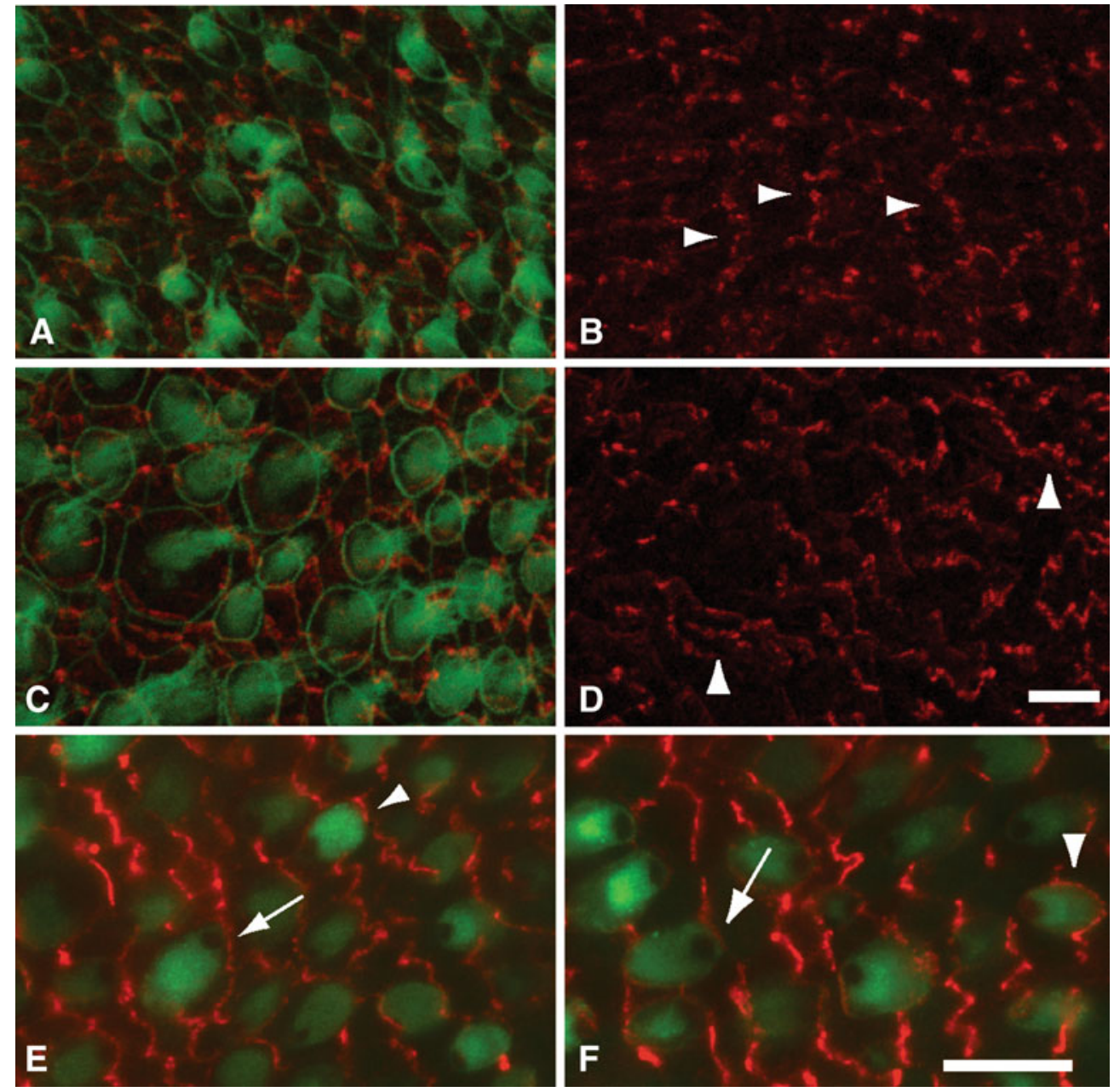

FIG. 5. Patterns of Vangl2 expression in the undamaged chick utricle. A-D Utricles were immunolabeled for Vangl2 (red), and hair cells and cell-cell junctions were labeled with phalloidin (green). All images are oriented such that the reversal zone is toward the right (i.e., in the coordinate system shown in Fig. 2A). Within the extrastriolar region (A, B), immunoreactivity for Vangl2 was observed at cell-cell junctions that were aligned with the reversal zone. Arrowheads in (B) point to strips of Vangl2 immunoreactivity that are approximately parallel to the reversal zone. In contrast, the reversal zone $(\mathbf{C}, \mathbf{D})$ frequently contained regions of Vangl2 expression that were perpendicular to the hair cell reversal line; examples are

the first to examine the expression of Vangl2 is the mature vertebrate ear. Since the chick inner ear is essentially mature and functional at the time of hatching, our results demonstrate that Vangl2 expression is maintained past the period of embryonic development. At present, it is not known whether the mature mammalian ear also continues to expresses Vangl2, but published data from the mouse cochlea suggest that Vangl2 expression levels begin to decrease by post-natal day 7 (Montcouquiol et al. 2006a). It is also notable that, in Drosophila, normal asymmetrical expression of the core PCP molecules (Fz, Dsh, Pk, and Vangl2) requires the expression of all other family members (e.g., Strutt

indicated by the arrowheads in (D). E, F Vangl2 expression at the reversal line. Hair cells were labeled with an antibody against $\alpha$ spectrin (green), which is expressed on the entire apical surface except for the insertion point of the kinocilium. We observed immunoreactivity for Vangl2 (red) adjacent to the kinociliar pole of some hair cells (arrows), as well as near the opposite side of other hair cells (arrowheads). However, because it was not possible to localize the Vangl2 immunoreactivity to a specific cell surface (hair cell vs. supporting cell), we could not determine whether the cellular localization of Vangl2 changed across the reversal line. Scale bars= $10 \mu \mathrm{m}$.

2008). As such, our observation of patterned expression of Vangl2 at cell-cell junctions in the mature avian ear suggests that the other PCP complex members also continue to be expressed.

The present study focused on the expression of Vangl2 within the medial extrastriolar region and the striolar reversal zone of the chick utricle (see Warchol and Speck 2007 for anatomical overview), and we did not closely examine the expression of Vangl2 in the (relatively small) lateral extrastriolar region. Expression of Vangl2 in the medial chick utricle is similar to the reported expression pattern of another core PCP molecule- $\mathrm{Pk} 2$-in the mouse utricle (Deans et al. 

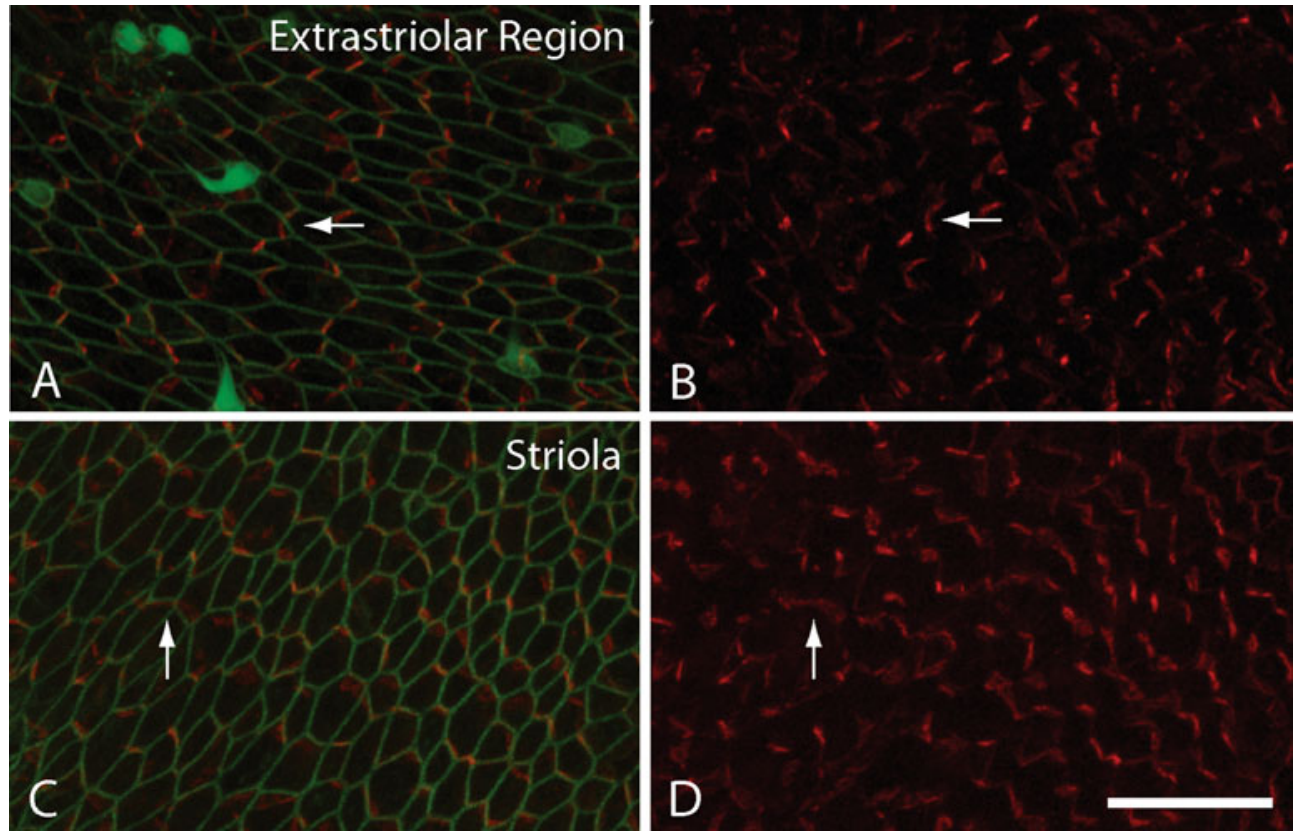

FIG. 6. Polarized expression of Vangl2 is maintained after severe ototoxic injury. Utricles were incubated for $24 \mathrm{~h}$ in $1 \mathrm{mM}$ streptomycin and then cultured for an additional $48 \mathrm{~h}$ in streptomycin-free medium. Cell-cell junctions and surviving stereocilia were labeled with phalloidin (green), and immunoreactivity for Vangl2 is shown in red. All images are oriented in the coordinate system

shown in Figure 2A. The extrastriolar region (A, B) contained a few surviving hair cells, and the expression of Vangl2 remained correctly polarized (B, arrow). The striolar region (C, D) contained no surviving hair cells, but Vangl2 expression was similar to that observed in unlesioned utricles and was often oriented perpendicular to the striola (D, arrow). Scale bar $=20 \mu \mathrm{m}$.
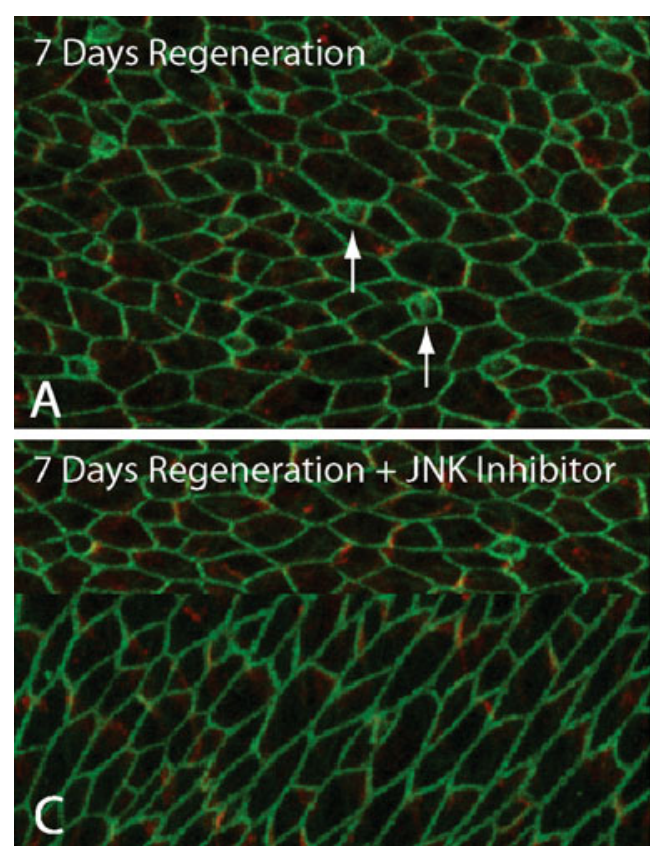

FIG. 7. A, B Maintained expression of Vangl2 during hair cell regeneration. Utricles were allowed to recover for 7 days after streptomycin injury and were then labeled with phalloidin (green) and anti-Vangl2 (red). Immature hair cells (arrows) were observed at 7 days after streptomycin treatment; normal patterns of Vangl2 expression were also observed. C, D Localization of Vangl2 after treatment with the JNK inhibitor SP600125. Utricles were treated
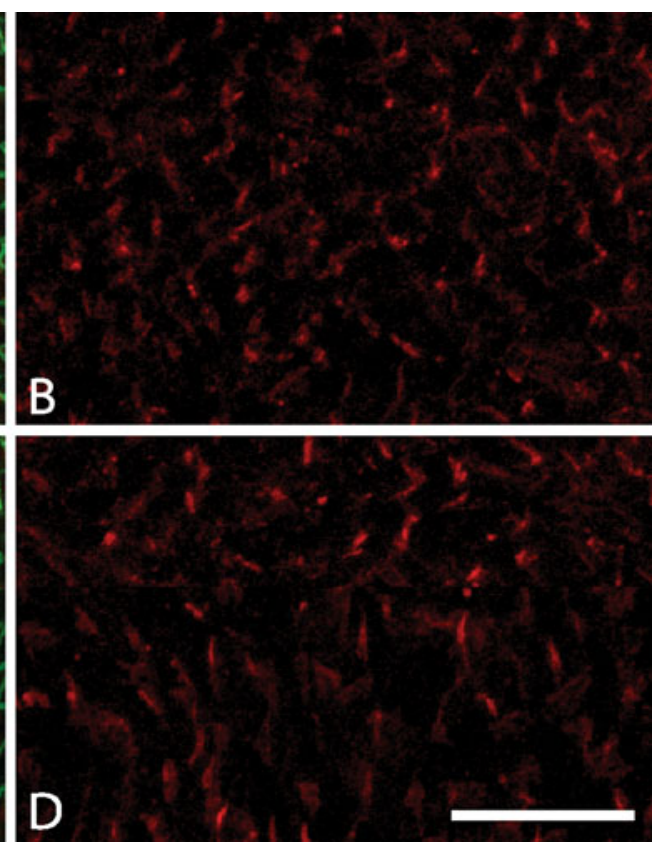

with streptomycin and then allowed to regenerate in vitro for 7 days. The JNK inhibitor SP600125 $(15 \mu \mathrm{M})$ was added to the cultures for the final 3 days of recovery. Although this treatment resulted in disarray of hair cell orientation (e.g., Fig. 8), inhibition of JNK did not cause changes in the patterned expression of Vangl2 (red) at cell-cell junctions (green, labeled with phalloidin). Scale bar $=20 \mu \mathrm{m}$. 

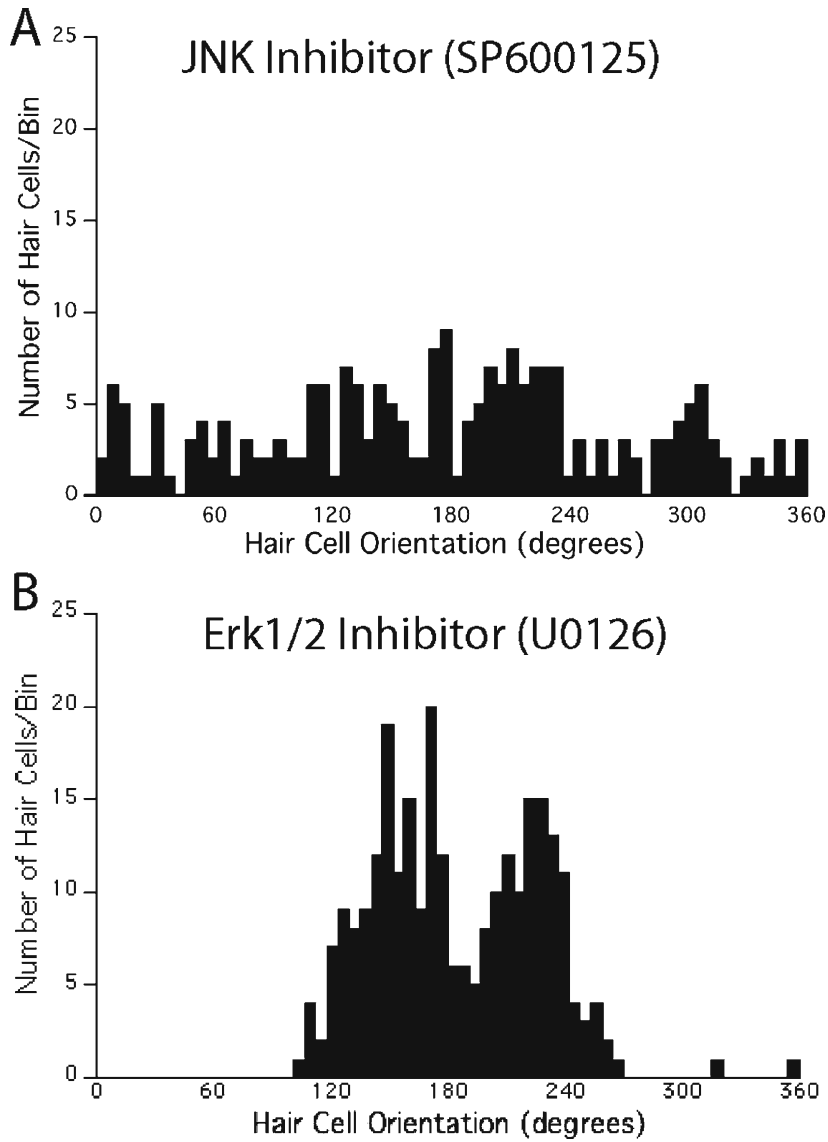

FIG. 8. Inhibition of JNK disrupts the orientation of regenerated stereocilia bundles. Utricles were treated for $24 \mathrm{~h}$ in $1 \mathrm{mM}$ streptomycin and then allowed to recover in vitro for 7 days. For the final $72 \mathrm{~h}$, the specimens also received SP600125 (15 $\mu \mathrm{M}$, a specific inhibitor of JNK, $n=10)$ or U0126 (10 $\mu \mathrm{M}$, a specific inhibitor of Erk1/2, $n=8$ ). After fixation and immunoprocessing, images of acetylated tubulin-labeled hair cells were obtained, and their angular orientations were quantified. A Histogram of the data obtained from hair cells $(n=223)$ in the SP600125-tretaed utricles shows a nearly random distribution of angular orientations. B Histogram of hair cell orientations $(n=264)$ obtained from the U0126-treated utricles shows a bimodal distribution that is centered at $\sim 180^{\circ}$. These data suggest that inhibition of JNKbut not Erk1/2 - severely disrupts the orientation of regenerating hair cells.

2007). Both PCP molecules are localized to cell-cell junctions that are generally orientated parallel to the anterior-posterior axis of the utricle. Surprisingly, cells on both sides of the reversal zone of the mouse utricle express $\mathrm{Pk} 2$ on their medial faces (Deans et al. 2007). This result appears to rule-out a direct role for $\mathrm{Pk} 2$ (and perhaps other core PCP molecules) in the establishment of the hair cell reversal line. Instead, the findings of Deans et al. (2007) are consistent with the notion that core PCP molecules specify the lateralmedial axis of the sensory epithelium. In the chick utricle, we found that the junctional orientation of Vangl2 is often shifted within the striolar reversal zone.
Although this result suggests that the regional expression of Vangl2 can change near the reversal line, it should also be noted that the anatomy of the reversal zone of the avian utricle is considerably different from its counterpart in the mammalian utricle. Specifically, the avian reversal zone is populated by an eight- to ten-cell-wide strip of type II hair cells, which can be recognized by their large apical surfaces (e.g., Jørgensen 1989) and expression of the GATA3 transcription factor (Warchol and Speck 2007). We found that the orientation of Vangl2 expression near such cells was often shifted by $\sim 90^{\circ}$ from that observed in neighboring regions (Fig. 5). The significance of this observation is unclear. A careful characterization of hair cell orientation within the reversal zone has not been carried-out, and it is possible that the observed localization of Vangl2 on these cells accurately reflects their polarization. We also attempted to determine whether the cellular localization of Vangl2 was similar to the expression of $\mathrm{Pk} 2$ in the mouse utricle (i.e., constant on both sides of the reversal line-Deans et al. 2007). Although our images are consistent with this notion (Fig. 5E,F), it was impossible to accurately determine which particular cell face (at a cell-cell junction) was the site of Vangl2 immunoreactivity. As such, determination of the cellular localization of Vangl2 must await study with immuoelectron microscopy.

One key observation of the present study is that asymmetric expression of Vangl2 is maintained in epithelial supporting cells after ototoxic injury. Since these cells serve as progenitors to replacement hair cells, it is reasonable to hypothesize that expression of Vangl2 can preserve cellular directionality after ototoxic injury and restore correct polarity via cell-cell contact. Previous studies have demonstrated that regenerated hair cells can be produced via two mechanisms: direct transdifferentiation and through renewed proliferation. A replacement hair cell that is created by direct transdifferentiation from a supporting cell could retain the expression of PCP molecules and use them to orient its stereocilia bundle. Most replacement hair cells, however, are produced by renewed proliferation. This fact raises the question of how the asymmetric expression of core PCP molecules can be transferred to both daughters of a dividing cell. One possibility is that the orientation of new epithelial cells is related to the orientation of the mitotic spindle of their "mother" cell, similar to a process that has been documented during Drosophila development (Bellaïche et al. 2001). To date, a single study has examined the possible relationship between the plane of cell division and the orientation of hair cell stereocilia. Live imaging of regeneration in lateral line neuromasts of zebrafish has revealed that pairs of oppositely oriented hair cells were produced by symmetric divisions of internal supporting cells (López-Schier and Hudspeth 2006). During that process, the plane of division was 
aligned with the neuromast's reversal zone; after division, each daughter cell then migrated to opposite sides of the neuromast. That study also showed that planar polarity in regenerated neuromasts was disrupted in fish that contained a mutated form of Vangl2 (trilobite). Although these results are conclusive for the model system under study (zebrafish lateral line), it is not clear how this mechanism would apply to the sensory organs of the inner ear. For one thing, many regenerated hair cells in the avian utricle arise via asymmetrical mitoses, which yield one hair cell and one supporting cell (Stone et al. 1999). In addition, all inner ear hair cells (except those in the macular reversal zones) have orientations that are very similar to those of neighboring hair cells. Based on the present results, we suggest that re-establishment of correct planar polarity in the vestibular maculae is guided by the maintained expression of core PCP molecules such as Vang12. We further predict that both daughter cells could inherit correct asymmetrical expression of PCP molecules, provided that the orientation of the mitotic spindle was perpendicular to the reversal line (rather than parallel, as in zebrafish neuromasts). Future studies should be able to use organotypic cultures, combined with time-lapse video microscopy, to resolve this issue.

\section{Role for JNK signaling in stereocilia orientation}

Although we observed nearly normal stereocilia orientation after regeneration in vitro, we also found that polarity was severely disrupted when regenerating cultures were treated with a small molecule inhibitor of the JNK. It is well established that JNK is a key signaling molecule in the noncanonical Wnt pathway (Axelrod and McNeill 2002; Montcouquiol et al. 2006b). Signaling in this pathway is initiated through the interaction of core PCP molecules, which are asymmetrically expressed on the membranes of opposing cells. Such interactions ultimately lead to the activation of JNK, which then acts on unknown targets to orient cells within epithelia. It is notable that genetic deletion of a number of "upstream" PCP molecules has been shown to result in defects in the alignment of stereocilia bundles (reviewed in Jones and Chen 2006), and it is likely that inhibition of JNK also blocks this pathway, leading to bundle misalignment. Finally, our observation that JNK inhibition does not alter Vangl2 expression (Fig. 7) suggests that the activity of JNK lies downstream of signaling by core PCP molecules.

\section{ACKNOWLEDGEMENTS}

Supported by Grants R01 DC006283 and P30 DC04665 from the NIDCD/NIH (MEW) and Avenir contract (MM).

\section{REFERENCES}

Axelrod JD, McNeill H (2002) Coupling planar polarity signaling to morphogenesis. Sci World 2:434-454

Bellaïche Y, Gho M, Kaltschimdt JA, Brand AH, Schweisguth F (2001) Frizzled regulates localization of cell-fate determinants and mitotic spindle rotation during asymmetric cell division. Nature Cell Biol 3:50-57

Bennett BL, Sasaki DT, Murray BW, O'Leary ED, Sakata ST, Xu W, Leisten JC, Motiwala A, Pierce S, Satoh Y, Bhagwat SS, Manning AM, Anderson DW (2001) SP600125: an anthrapyrazolone inhibitor of Jun N-terminal kinase. Proc Natl Acad Sci USA 98:13681-13686

Boutros M, Paricio N, Strutt Di, Mlodzik M (1998) Dishevelled activates JNK and discriminates between JNK pathways in planar polarity and wingless signaling. Cell 94:109-118

Dabdoub A, Kelley MW (2005) Planar cell polarity and a potential role for a Wnt morphogen gradient in stereocilia bundle orientation in the mammalian inner ear. J Neurobiol 64:446457

Dabdoub A, Donohue MJ, Brennan A, Wolf V, Montcouquiol M, Sassoon DA, Hsein J-C, Rubin JS, Salinas PC, Kelley MW (2003) Wnt signaling mediates reorientation of outer hair cell stereociliary bundles in the mammalian cochlea. Development 130:2375-2384

Davies A, Formstone C, Mason I, Lewis J (2005) Planar polarity of hair cells in the chick inner ear is correlated with polarized distribution of c-flamingo-1 protein. Dev Dyn 233:998-1005

Deans MR, Antic D, Suyama K, Scott MP, Axelrod JD, Goodrich LV (2007) Asymmetric distribution of prickle-like 2 reveals an early underlying polarization of vestibular sensory epithelia in the inner ear. J Neurosci 27:3139-3147

Dye BJ, Frank TC, Newlands SD, Dickman JD (1999) Distribution and time course of hair cell regeneration in the pigeon utricle. Hearing Res 133:17-26

Favata MF, Horiuchi KY, Manos EJ, Daulerio AJ, Stradley DA, Feeser FS, Van Dyk DE, Pitts WJ, Earl RA, Hobbs F, Copland RA, Magolda RL, Scherle PA, Trzaskos JM (1998) Identification of a novel inhibitor of mitogen-activated protein kinase kinase. J Biol Chem 273:18623-18632

Jones C, Chen P (2006) Planar cell polarity signaling in vertebrates. BioEssays 29:120-132

JøRGENSEN JM (1989) Number and distribution of hair cells in the utricular maculae of some avian species. J Morphol 201:187204

Kim GH, HAN JK (2005) JNK and ROKalpha function in the noncanonical Wnt/RhoA signaling pathway to regulate Xenopus convergent extension movements. Dev Dyn 232:958-968

LÓPEZ-SCHIER H, HudSPETH AJ (2006) A two-step mechanism underlies the planar polarization of regenerating sensory hair cells. Proc Natl Acad Sci USA 103:18615-18620

Lysakowski A, Goldberg JM (2005) Morphophysiology of the vestibular periphery. In: Highstein SM, Fay RR, Popper AN (eds) The vestibular system. Springer, New York

Matsui Ji, Oesterle EC, Stone JS, Rubel EW (2000) Characterization of damage and regeneration in cultured avian utricles. J Assoc Res Otolaryngol 1:46-63

Matsui Ji, Parker MA, Ryals BM, Cotanche DA (2005) Regeneration and replacement in the vertebrate inner ear. Drug Discov Today 10:1307-1312

Montcouguiol M, Rachel RA, Lanford PJ, Copeland NG, Jenkins NA, KeLLEY MW (2003) Identification of Vang12 and Scrb1 as planar polarity genes in mammals. Nature 423:173-177

Montcouguiol M, Sans N, Huss D, Kach J, Dickman JD, Forge A, Rachel RA, Copeland NG, Jenkins nA, Bogani D, Murdoch J, Warchol ME, Wendhold RJ, Kelley MW (2006A) Asymmetric 
localization of Vang12 and Fz3 indicate novel mechanisms for planar polarity in mammals. J Neurosci 26:5265-5275

Montcouquiol M, Crenshaw EB 3Rd, Kelley MW (2006в) Noncanonical Wnt signaling and neural polarity. Ann Rev Neurosci 29:363-386

Stone JS, Cotanche DA (2007) Hair cell regeneration in the avian auditory epithelium. Int J Dev Biol 51:633-647

Stone JS, Choi YS, Woolley SMN, Yamashita H, Rubel EW (1999) Progenitor cell cycling during hair cell regeneration in the vestibular and auditory epithelia of the chick. J Neurocytol 28:863-876

StRUTT D (2008) The planar polarity pathway. Curr Biol 18:R898-R902

Wang Y, Guo N, Nathans J (2006) The role of Frizzled3 and Frizzled6 in neural tube closure and in the planar polarity of inner-ear sensory hair cells. J Neurosci 26:2147-2156

WARCHOL ME (1999) Immune cytokines and dexamethasone influence sensory regeneration in the avian vestibular periphery. J Neurocytol 28:889-900
WARCHOL ME (2001) Lectin from Griffonia simplicifolia identifies and immature-appearing subpopulation of sensory hair cells in the avian utricle. J Neurocytol 30:253-264

Warchol ME, Richardson GP (2009) Expression of the Pax2 transcription factor is associated with vestibular phenotype in the avian inner ear. Dev Neurobiol 69:191-202

WARCHOL ME, SPECK JD (2007) Expression of GATA3 and tenascin in the avian vestibular maculae: Normative patterns and changes during sensory regeneration. J Comp Neurol 500:646-657

Weisleder P, Rubel EW (1993) Hair cell regeneration after streptomycin toxicity in the avian vestibular system. J Comp Neurol 331:97-110

WolfF T, Rubin GM (1998) Strabismus, a novel gene that regulates tissue polarity and cell fate decisions in Drosophila. Development 125:1149-1159

ZAKIR M, DickMAn JD (2006) Regeneration of vestibular otolith afferent after ototoxic damage. J Neurosci 15:2881-2893 